\title{
Mathematical Representation of the Velocity, Temperature and Solid Fraction in an Electromagnetically Stirred Solidifying Melt
}

\author{
Olusegun Johnson ILEGBUSI and Julian SZEKELY \\ Department of Materials Science and Engineering, Massachusetts Institute of Technology, Cambridge, MA 02139, U.S.A.
}

(Received on September 4, 1989; accepted in the final form on January 26, 1990)

\begin{abstract}
A mathematical model has been developed to describe the velocity, temperature and solid fraction in an electromagnetically stirred $\mathrm{Al}-5 \% \mathrm{Cu}$ alloy in which $15 \% \mathrm{~B}_{4} \mathrm{C}$ particles were initially suspended. In the model, we allowed for the rheology of the melt-solid slurry and the release of latent heat either at the melt-solid interface, in the bulk or at an intermediate stage between those extremes. The calculations have shown that the evolution of the temperature and solid fraction profiles were not significantly affected, either by the stirring rate or by the choice of the location of release of latent heat.
\end{abstract}

KEY WORDS: mathematical modeling; electromagnetism; agitation; solidification.

\section{Introduction}

In recent years there has been a growing interest in the study of "rheocasting" and "compocasting", which essentially involve the solidification of strongly agitated melts under conditions such that part of the two-phase region is being detached from the advancing solidification front. The concept of rheocasting was first put forward by Flemings and Mehrabian, ${ }^{1)}$ who suggested that interesting, very desirable solid structures may be produced when a binary or multicomponent melt is strongly agitated during the solidification process. Under these conditions, the growth of the solid phase takes place both in the bulk and through the advancement of the solidification front. The perceived attraction of rheocasting is that segregation may be minimized and that rather finer grain structures may be produced than by conventional means.

A perhaps less extreme step in this direction is provided by the conventional electromagnetic stirring of steel in continuous casting, which is thought to promote equiaxed structures. ${ }^{2,3)}$ This is also the case in the recently developed CREM process ${ }^{4)}$ where grain refinement is being produced by strong stitrring of the melt during solidification in the production of $\mathrm{DC}$ cast aluminum ingots.

Yet another variation on this theme is "compocasting" where a melt containing suspended solid particles (e.g., boron carbide in aluminum) is being solidified, while providing strong agitation. Here solid phases are being provided by both the originally present suspended solid particles and the nucleating solidified material.

An excellent review of the recent works on the physical and mathematical modeling of solidification has been presented by Rapaz. ${ }^{5}$ Most of the work done to date on the solidification of rheocast or com- pocast materials, e.g., ${ }^{6,7)}$ has concentrated on the characterization of the solidified structures, rather than on modeling the transport phenomena in the process itself. The modeling effort has been largely concentrated on heat transfer, rather than on the combined effects of heat transfer and fluid flow.

As discussed in earlier publications by the authors, ${ }^{8,9)}$ the modeling of fluid flow phenomena in melt-solid slurries is complicated by the fact that these systems exhibit strongly non-Newtonian behavior and by the paucity of information on the rheological behavior of these systems. Nonetheless, it is generally appreciated that these melt-solid slurries exhibit shear-thinning characteristics, so that if a high enough shear rate or stirring power is being applied, they will remain relatively free flowing even at high solids loading levels.

Ultimately in the design of laboratory scale apparatus and that of industrial scale equipment for rheocasting or compocasting, we wish to relate the stirring power, the stirring arrangements and the cooling rates to the velocity and the temperature fields in the melt. The final objective will be to develop an explicit relationship between these parameters and the solid structures that are being produced.

The present paper is intended as a first effort in trying to combine the recently developed ability to calculate the velocity fields in agitated melt-solid slurries with the laws of solidification. The main objective of this work, at this stage, is to provide a sense of perspective of the role played by some of the key process parameters in defining the progress of solidification in a laboratory type rheocasting apparatus.

\section{Formulation-statement of the Problem}

Let us consider a cylindrial vessel, sketched in 


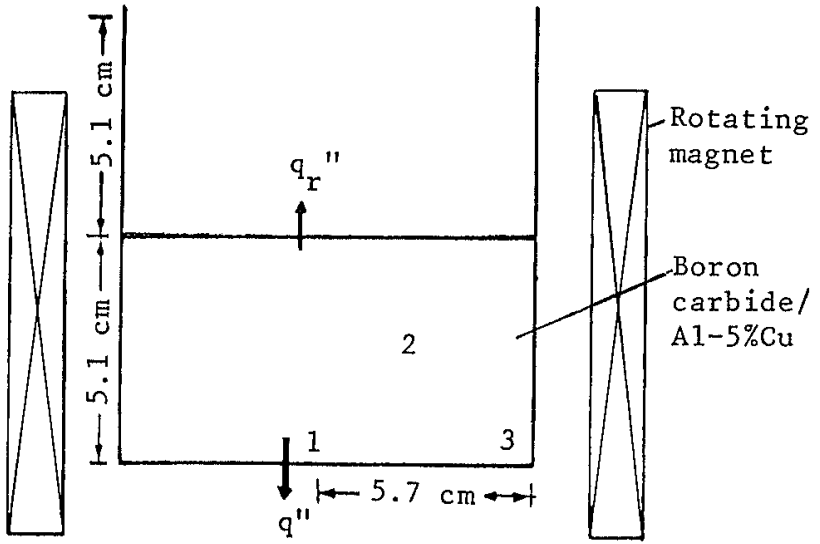

Fig. 1. A schematic sketch of the system considered including the three points 1, 2, 3 to be monitored.

Fig. 1 which contains an initially molten alloy, in which inert solid particles are being suspended. The material chosen for the calculations was an Al$5 \% \mathrm{Cu}$ alloy in which boron carbide particles (15 $\mathrm{wt} \%$ ) were being suspended.

This melt is being agitated by a rotating electromagnetic field, of the type described in a previous publication. ${ }^{9}$ The side walls of the vessel are insulated and the bottom surface is being cooled. The top surface is allowed to radiate to the environment, thus the effective cooling at the top surface is much less than that at the bottom.

The following principal assumptions are being made:

(a) The system is axi-symmetric.

(b) The free surface of the melt is assumed to be flat and impervious to fluid flow. In reality, there will be a vortex formation as a result of the fluid rotation. However, the assumed flat surface simulates a system with frictionless wall.

(c) Flow in the melt may be described by the two dimensional Navier-Stokes equations, with an appropriate shear-thinning model to represent the effective viscosity of the melt. Thus the melt viscosity will depend on the solid fraction and on the shear rate. Since the shear rate is both direction and position dependent, this relationship will be quite complex.

(d) As far as heat transfer and solidification are concerned, a continuum type approach has been taken with an emphasis on predicting the temperature profiles and the time dependent position of the solidification front. In rheocast systems, heat transfer phenomena are generally complicated by the fact that solidification and the release of the latent heat will take place both in the bulk (giving rise to a meltsolid suspension) and at the melt-solid boundary. The actual partitioning of the latent heat release between these two regions will have to depend on the extent of agitation. With zero agitation, most of the two phase region will adhere to the continuum solidification boundary, while for strong agitation much of the latent heat may be released in the bulk. For the present, this partitioning has been regarded as a parameter. The sensitivity of the results to the values chosen for this parameter will be explored as part of the study.

\subsection{Governing Equations}

Within the framework of these assumptions, the governing equations may be put in the following form:

Gontinuity Equation:

$$
\frac{\partial w}{\partial z}+\frac{1}{r} \frac{\partial r v}{\partial r}=0
$$

Axial Momentum:

$$
\frac{\partial \rho w}{\partial t}+\nabla \cdot(\rho U w)=-\frac{\partial p}{\partial z}-(\nabla \cdot \tau)_{z}+F_{z}
$$

Radial Momentum:

$$
\frac{\partial \rho v}{\partial t}+\nabla \cdot(\rho U v)=-\frac{\partial p}{\partial r}-(\nabla \cdot \tau)_{r}+F_{r}
$$

Azimuthal Momentum:

$$
\frac{\partial \rho u}{\partial t}+\nabla \cdot(\rho U u)=-(\nabla \cdot \tau)_{\theta}+F_{\theta}
$$

In the above equations,

$p:$ the static pressure

$\tau:$ the shear stress tensor

$F=\left(F_{r}, F_{\theta}, F_{z}\right):$ the electromagnetic body force

the subscripts: components in the corresponding coordinate directions.

The actual expressions for the body forces and the shear stress will be presented in a subsequent section.

\section{Thermal Energy Balance:}

The conservation of thermal energy can be conveniently expressed in terms of the enthalpy $h\left(=C_{p} T\right)$ in the form:

$$
\rho \frac{\partial h}{\partial t}+\rho U \cdot \nabla h=\nabla \cdot(k \nabla h)+S_{h}
$$

where, $k$ : the thermal diffusivity

$S_{h}$ : the enthalpy source term.

It should be noted that this enthalpy method has been applied to relatively simpler problems than that considered here, by Voller and co-workers. ${ }^{10,11)}$ The present formulation defers from the latter however, in the method used to calculate $S_{h}$ as will be described below.

\subsection{Auxiliary Relations}

\subsubsection{The Latent Heat Source}

By writing Eq. (5) in terms of the total enthalpy $H(=h+\Delta H)$, in which $h$ is the sensible enthalpy and $\Delta H$ is the latent heat, we can express the enthalpy source $S_{h}$ in the form:

$$
S_{h}=\rho \frac{\partial \Delta H}{\partial t}-\nabla \cdot(\Delta H \rho U)
$$

The method of determining $\Delta H$ will be described below.

In contrast to the solidification of one component systems, the present problem iñvölves a binary alloy 
solidifying in contact with an agitated melt-solid slurry. Thus, part or the whole of the mushy zone may not attach to the solidifying surface due to the melt motion. A direct consequence of this is that only part of the latent heat will be absorbed at the advancing solidification boundary while the remainder will have to be absorbed in the bulk of the melt.

In order to account for these effects, we postulate that the solid surface will be at a temperature $T_{r}$ between the liquidus and solidus temperatures and that a portion $L_{r}$ of the latent heat would be absorbed at this interface. The remainder of the latent heat would thus be released in the melt. The latter is accounted for by an increase in the effective specific heat $C_{p \text { eff }}$ in the bulk by the following expression:

$$
C_{p \text { eff }}= \begin{cases}C_{p l}+\frac{L-L_{r}}{T_{l}-T_{r}}=C_{p l}+\frac{L}{T_{l}-T_{s}} & T \leq T_{l} \\ C_{p l} & \mathcal{T}>T_{l}\end{cases}
$$

where, $\quad C_{p l}$ : the specific heat of the melt

$L:$ the latent heat of fusion

$T_{l}, T_{s}$ : the liquidus and solidus temperatures, respectively.

Eq. (7) also implies that by choosing $T_{r}$ as a parameter, we can determine $L_{r}$ from the relationship:

$$
L_{r}=L T^{*}
$$

where $T^{*}$ is the proportion of the mushy region that adheres to the surface expressed as:

$$
T^{*}=\frac{\left(T_{r}-T_{s}\right)}{\left(T_{l}-T_{s}\right)}
$$

A more detailed discussion of this approach has been provided by Stanek and Szekely. ${ }^{12}$ )

In view of the above, the enthalpy source can thus be expressed in the form:

$$
\Delta H= \begin{cases}0 & T<T_{r}, T>T_{l} \\ \Delta h & T_{r} \leq T \geq T_{l}\end{cases}
$$

where:

$$
\Delta h= \begin{cases}L_{r}+\frac{\left(L-L_{r}\right)\left(T-T_{r}\right)}{\left(1-T^{*}\right)\left(T_{l}-T_{s}\right)} & T_{s} \leq T_{r}<T_{l} \\ L & T_{r}=T_{l}\end{cases}
$$

\subsubsection{Shear Stress Tensor}

We shall assume that the semi-slurry system obeys a power-law relationship thus:

$$
\tau=-\left[m \mid\left(\left.0.5(\gamma: \gamma)^{1 / 2}\right|^{n-1}\right] \gamma\right.
$$

where $\gamma$ is the rate of deformation tensor, $m$ (the fluid consistency) and $n$ are empirical constants which we define by the expressions:

$$
\begin{aligned}
& m=\exp \left(9.783 f_{s}+1.4345\right) \\
& n= \begin{cases}0.1055+0.41 f_{s} & 0.15 \leq f_{s}<0.3 \\
-0.308+1.78 f_{s} & 0.30 \leq f_{s}\end{cases}
\end{aligned}
$$

in which $f_{s}$ is the solid fraction.

It should be pointed out the above relationships were derivatives of those deduced for a $\mathrm{Pb}-\mathrm{Sn}$ system by Laxmanan. ${ }^{7)}$ It is not immediately obvious whether the relationships will hold for the $\mathrm{Al}-\mathrm{Cu}$ system considered in the present study. However, it is felt that the general nature of these systems, particularly the shear-thinning behavior, would be fairly well represented by them.

\subsubsection{Electromagnetic Force}

The electromagnetic force components $F_{r}, F_{0}$ and $F_{z}$ appearing in Eqs. (2)-(4) are evaluated through the Maxwell's equations. In the present system, we assume an applied rotating magnetic field that is sinusoidal in time and angular coordinates. The force components for a single-pole magnet employed here are approximately:

$$
\begin{aligned}
& F_{z}=0 \ldots \ldots \ldots \ldots \ldots \ldots \ldots \ldots \ldots \ldots \ldots \\
& F_{r}=-\frac{1}{8} B_{0}^{2}\left(\omega-\frac{u}{r}\right)^{2} \sigma^{2} \mu_{0} r^{3} \\
& F_{o}=\frac{1}{2} B_{0}^{2}\left(\omega-\frac{u}{r}\right) \sigma r
\end{aligned}
$$

where, $\omega$ : the angular velocity of the field

$B_{0}$ : applied magnetic field of the outer surface $\sigma:$ the electrical conductivity

$\mu_{0}$ : the magnetic permeability.

The derivation of Eqs. (14)-(16) is detailed in Spitzer et $a l .{ }^{13)}$ and will not be repeated here.

\subsection{Boundary Conditions}

The boundary conditions employed in the computations are:

i) No-slip at all walls

ii) Symmetry about the centerline

iii) No stress acts at the free surface

iv) Thermal radiation to the environment at the free surface

v) Insulation at side walls

vi) Heat loss at the bottom is determined by a Newtonian exchange with a surrounding at temperature $T_{e}$ in the form:

$$
q=h_{c}\left(T_{i}-T_{e}\right)
$$

where, $q$ : the rate of heat loss per unit area

$h_{c}$ : the heat transfer coefficient

$\tau_{i}$ : the temperature of the material in contact with the cooled surface.

In essence, these governing equations and boundary conditions are quite standard for work of these types. However, comments should be made on the following two aspects:

First, in the statement of the solidification problem, we are making an allowance for two possible limiting cases, namely:

a) When all the mushy zone remains attached to the solidified region, which corresponds to the latent heat release in the two phase zone, i.e., $T^{*}=1$

b) When all the solidification occurs within the melt, 
through the growth of crystals suspended in the melt, in which case all the latent heat is released in the bulk of the melt, i.e., $T *=0$,

c) When half the latent heat is being released at the melt-solid boundary, while the remainder has to be absorbed by the bulk of the melt, i.e., $T^{*}=$ 0.5 .

Strictly speaking, the proper representation of these problems require a nucleation-growth model. These issues will be considered in a subsequent paper.

The second point that needs emphasis is that as solidification proceeds in the bulk of the melt, the solid fraction will increase and hence the effective viscosity of the melt-solid slurry will also increase. This aspect of the problem is represented by the model presented here.

\section{Method of Solution}

The above governing equations were cast in finite domain forms and solved with the PHOENICS code. $^{14)}$ A spatially non-uniform $28 \times 18 \times 36$ grid structure was used in the radial, azimuthal and axial directions respectively. A fully implicit scheme which is unconditionally stable was employed. The results were found to be grid independent in both the spatial and temporal coordinates and took about $12 \mathrm{~h}$ of GPU time for $80 \mathrm{~s}$ of real time on a Micro Vax II computer.

\section{Computed Results}

In the following we shall present a selection of the computed results, showing the evolution of the velocity profiles, temperature profiles and maps of the solid fraction in the system.

In a qualitative sense, one may map out " $\mathrm{ex}-$ pected behavior" of this system.

In the absence of magnetic field, one may expect very low melt velocities, driven by natural convection only and an essentially flat solid fraction 'profile, which will depend on time, but not on the radial position in the system.

When agitation is being applied, the behavior of the system will necessarily depend on the boundary condition imposed, regarding the release of the latent heat. If we assume that the latent heat is released predominantly at the melt-solid boundary, one would expect a relatively rapid growth of the fully solidified layer first, with a gradual slow-down as the solidified shell represents an increased resistance to heat flow. The converse will be true, if the latent heat is assumed to be released in the bulk. Here the solidified shell will grow very slowly in the initial stages, as the solid fraction is being built up in the bulk. However, the growth of the solidified shell will accelerate for subsequent times. One may also expect that for an identical stirring force, the velocity in the melt will decrease with the build up of the solid fraction in this latter case.

These arguements appear logical, however, the extent to which this behavior is being observed in a quantitative manner can be established through numerical analysis.

The actual input parameters used in the computation are summarized in Table 1. It is seen that we have considered an $\mathrm{Al}-\mathrm{Cu}$ alloy, in which $10 \%$ by weight of boron carbide has been suspended.

Fig. 2 shows a typical plot of the velocity vectors in the horizontal plane $(z / H=0.5)$ at some intermediate time $t=40 \mathrm{~s}$. The general nature of the rotating melt is readily apparent.

Fig. 3 shows the evolution of the absolute value of the maximum melt velocity with time, in the absence of magnetic field, i.e., in the absence of forced flow. Here we assumed that all the latent heat is released at the melt-solid boundary, i.e., $T^{*}=1$. It is seen that the velocity due to natural convection will be very small. The velocity plot corresponding to point I shows an increase with time and then quite a rapid decrease is being observed. This behavior is logical, because point 1 is located at the center of the cooled plane, thus the cessation of velocity corresponds to the establishment of a solidification front at that location. The velocity at point 2 begins to decline after about $60 \mathrm{~s}$, as the solidification front approaches it.

Fig. 4 shows the absolute value of the maximum velocity plots corresponding to the same locations but

Table 1. Principal input parameters used in the computation.

\begin{tabular}{ll}
\hline Melt height & $5.1 \mathrm{~cm}$ \\
Melt radius & $5.7 \mathrm{~cm}$ \\
Melt material & $\mathrm{AI}-5 \% \mathrm{Cu}$ \\
Particles & $\mathrm{B}_{4} \mathrm{C}$ \\
Initial concentration of particles & $15 \%$ \\
Heat transfer coefficient $\left(h_{\mathrm{c}}\right)$ & $1000 \mathrm{Wm}^{-2} \mathrm{~K}^{-1}$ \\
Surrounding temperature $\left(T_{e}\right)$ & $298 \mathrm{~K}$ \\
Latent heat & $3.7 \times 10^{5} \mathrm{~J} \mathrm{~kg}^{-1} \mathrm{~K}^{-1}$ \\
Magnetic fields considered & $0,1000,2500 \mathrm{Gauss}^{-1}$ \\
Angular velocity of fields & $600 \mathrm{rad} / \mathrm{s}$ \\
Solidus temperature & $860 \mathrm{~K}$ \\
Liquidus temperature & $920 \mathrm{~K}$ \\
Initial temperature & $933 \mathrm{~K}$ \\
Electrical conductivity & $1.33 \times 10^{6} \Omega^{-1} \mathrm{~m}^{1}$
\end{tabular}

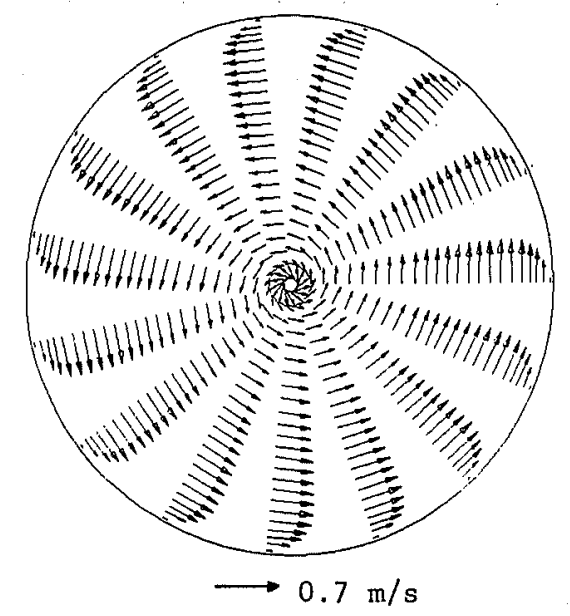

Fig. 2. Velocity vectors in the horizontal plane for $B=1$ kGauss and $t=40 \mathrm{~s}$. 


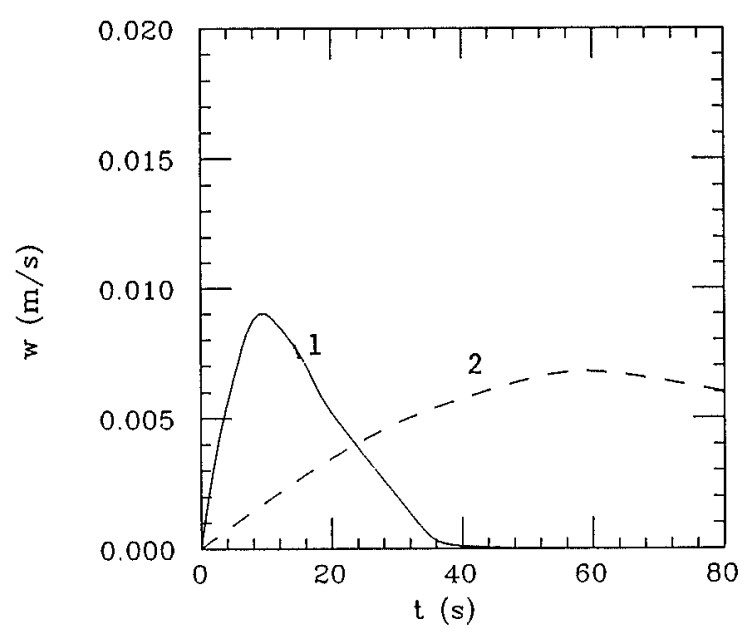

Fig. 3. Evolution of maximum velocity in the absence of magnetic field.

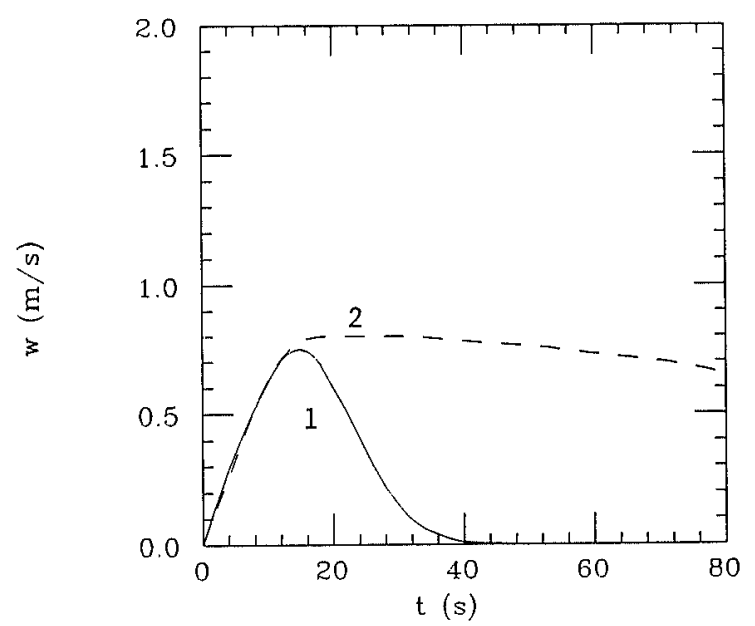

Fig. 4. Evolution of the maximum velocity with magnetic field of 1 kGauss.

for stirring with a rotating field of 1 kGauss. In this case, the latent heat of solidification was assumed to be released partly in the bulk ( $5 \%$ ) and partly at the melt-solid interface, i.e., $T^{*}=0.5$.

The absolute values of the velocity are of course much higher; the velocity at point 1 reaches its maximum over a relatively short time period and its decline corresponds again to the approaching solidification front.

Fig. 5 shows the effect of stirring rate (more precisely that of the magnetic field, which is approximately proportional to the velocity) on the evolution of the temperature at the central point of the cooled plane (point 1). In the computation of these curves, we assumed $\left(T^{*}=0\right)$, i.e., the latent heat being released in the bulk.

It is seen that point $l$ will cool the fastest, when the least stirring is being applied. This behavior is consistent with physical reasoning, as discussed before.

Fig. 6 shows the corresponding plots, but at a location 2, i.e., in the bulk of the system. It is seen that here the behavior is a reverse of that observed in Fig. 5 , in that higher agitation will provide a more rapid cooling.

Fig. 7 shows the advancement of the solidification

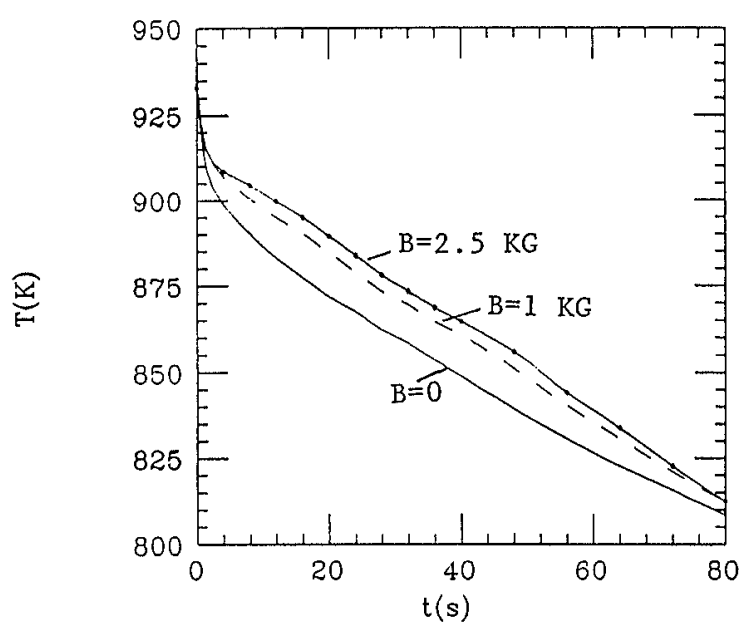

Fig. 5. Effect of stirring rate $(B)$ on the evolution of temperature at the central point (1) of the cooled plane.

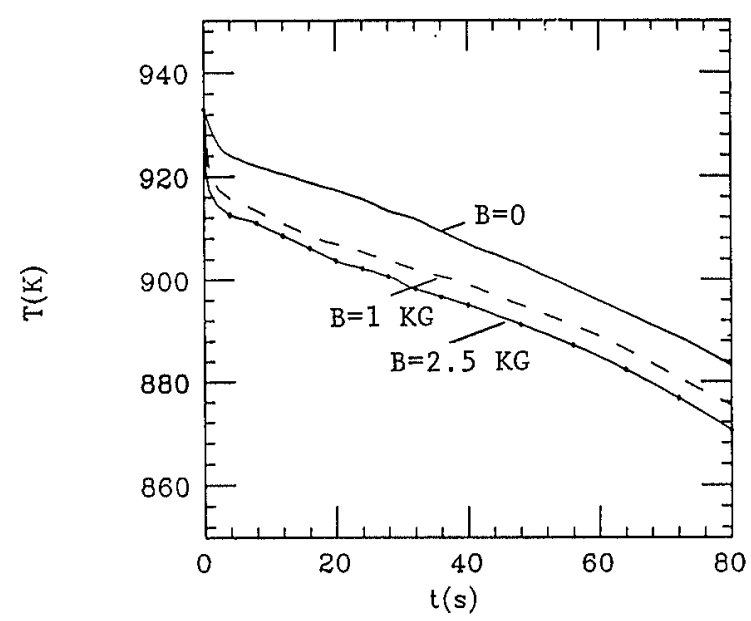

Fig. 6. Effect of stirring rate $(B)$ on the evolution of temperature at point 2 located in the bulk of the melt.

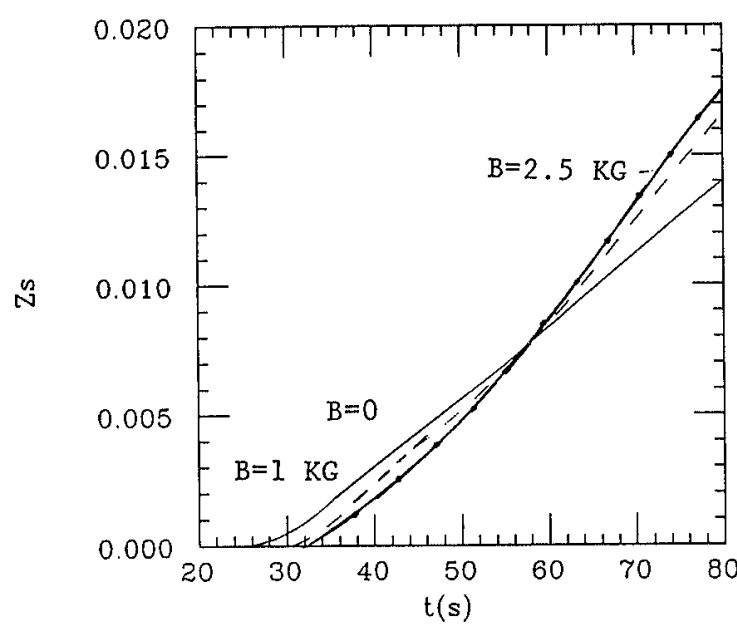

Fig. 7. Effect of stirring rate $(B)$ on the advancement of the solidification front at point 3 located near the wall corner on cooled plane.

front for a case, where all the latent heat is released at the melt-solid boundary, for various stirring conditions. This plot refers to position 3 at the cooled horizontal plane near the wall corner. It is seen that in the initial stages the solidification front will advance more rapidly when no stirrirs is being applied, but 
that a "switchover" will occur after about $60 \mathrm{~s}$. This trend is consistent with the results presented in Figs. 5 and 6.

Fig. 8 depicts the behavior of point 1 at the center of the cooled plane. It is seen that Figs. 7 and 8 are qualitatively similar.

Fig. 9 shows the evolution of the solid fraction at the central plane. The solid fraction resulting from the solidifying melt is calculated from the relation:

$$
f_{s s}=1-\Delta H / L
$$

However, the solid fraction $f_{s}$ plotted in Fig. 9 and subsequent figures has to take account of the initial solid concentration $f_{s 0}$ which is assumed to be uniform in the melt. Thus, $f_{s}$ is calculated from the relation:

$$
f_{s}=\min \left(f_{s s}+f_{s 0}, 1.0\right)
$$

It is seen that the solid fraction will evolve more rapidly, the less the agitation.

The following plots illustrate the effect of allowing the release of the latent heat to occur in the bulk $\left(T^{*}=0\right)$, in the mushy zone $\left(T^{*}=1\right)$ or in a manner equally divided between these two regions $\left(T^{*}=0.5\right)$. All the plots refer to a stirring rate corresponding to

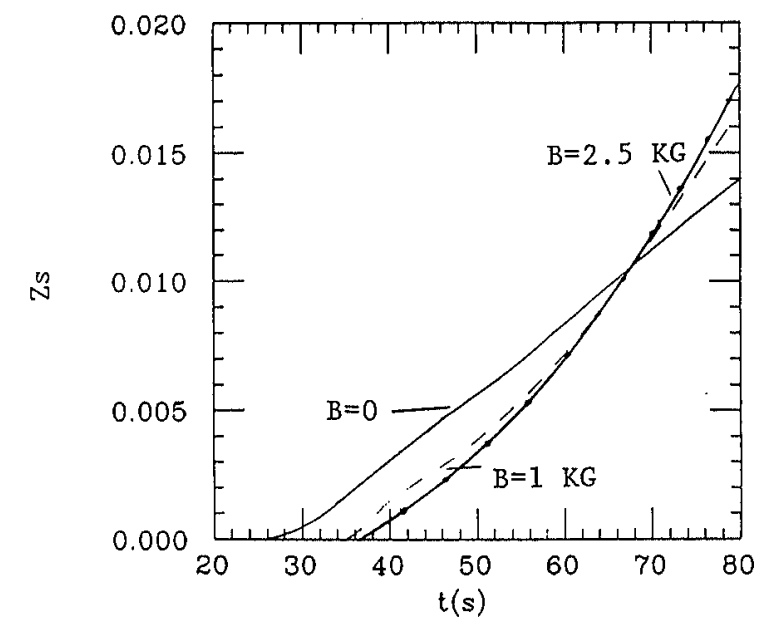

Fig. 8. Effect of stirring rate $(B)$ on the advancement of the solidification front at point 1 .

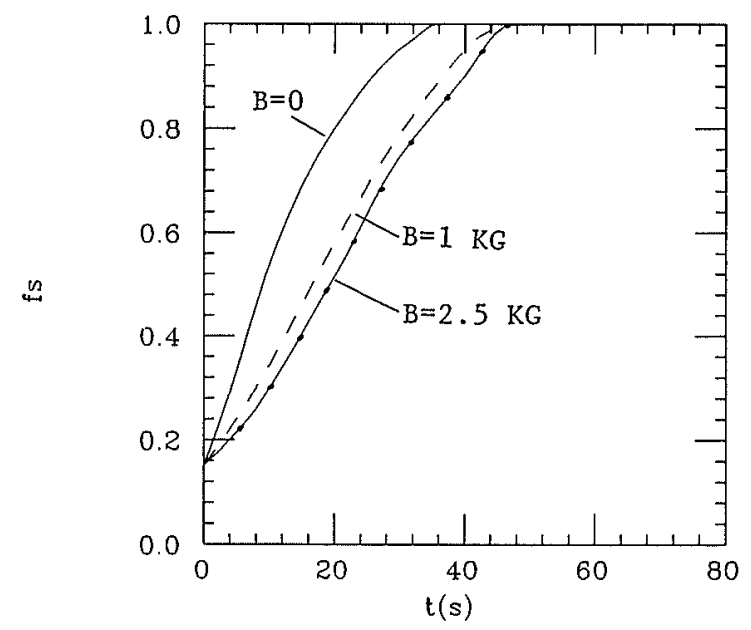

Fig. 9. Effect of stirring rate on evolution of solid fraction at point 1 .

\section{1 kGauss.}

Fig. 10 shows the evolution of the temperature at point 1 and this behavior is consistent with that seen previously in Fig. 5. Fig. 11 shows a corresponding plot, but now at point 2 , i.e., in the bulk of the melt. It is seen that the behavior is also qualitatively similar to Fig. 10, but that the temperatures are of course higher, some distance away from the cooled surface.

Figs. 12 and 13 show the evolution of the solidificatian front with the value of $T^{*}$ as a parameter. It is seen that the solidification front will advance the fastest, when the latent heat is being released in the mushy region. The behavior at the central point 1 (Fig. 12) is observed to be quite similar to that of the near-wall point 3 shown in Fig. 13. It should be stressed that in a sense these plots are artificial, because one cannot specify $T^{*}$ a priori; rather, the plots represent the bounds of behavior that may be expected.

Finally, Figs. 14(a) to 14(d) show the maps of the solid fraction at different times, both in the absence and in the presence of stirring. These plots are helpful, because they provide a global view of how the solidification process progresses with time. It is seen that in the absence of forced flow, the solid fraction

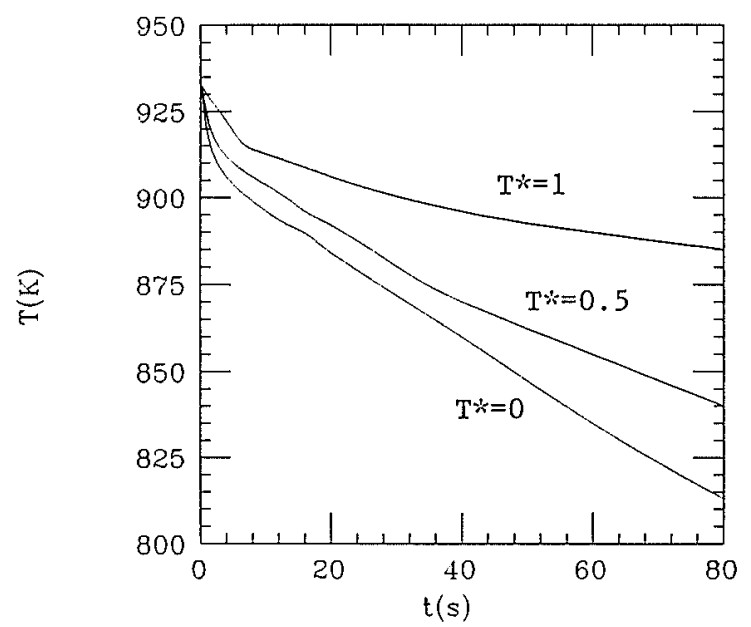

Fig. 10. Evolution of temperature at point 1 for $B=$ $1 \mathrm{kGauss}$ with $T^{*}$ as a parameter.

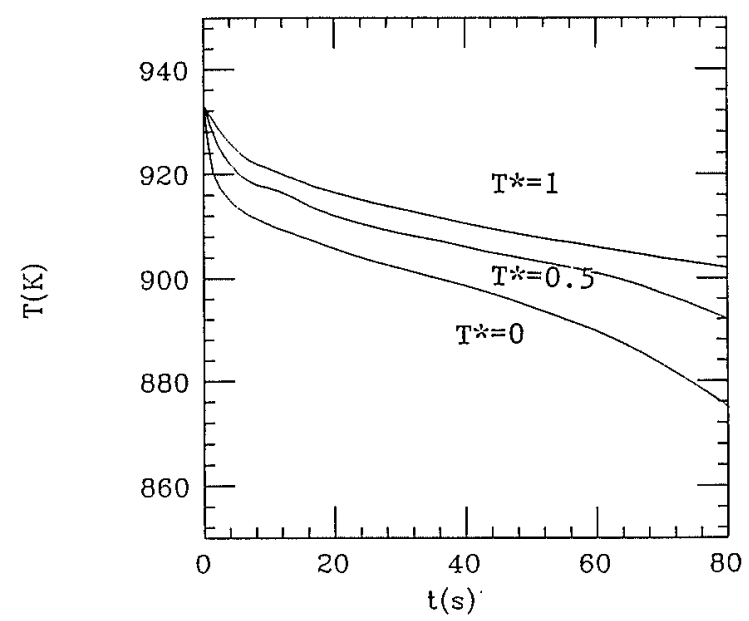

Fig. 11. Evolution of temperature at point 2 for $B=$ $1 \mathrm{kGauss}$ with $T^{*}$ as a paramèter. 


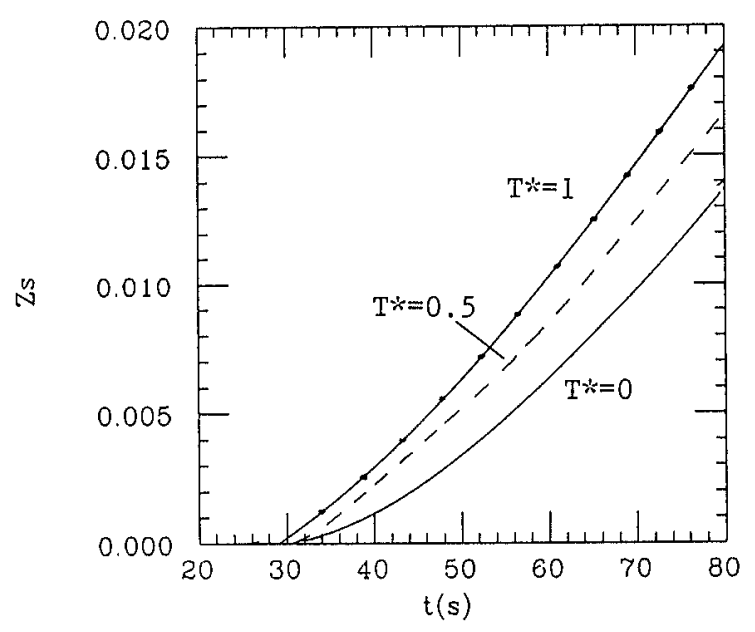

Fig. 12. Evolution of solidification front at point 1 for $B=$ $1 \mathrm{kGauss}$ with $T^{*}$ as a parameter.

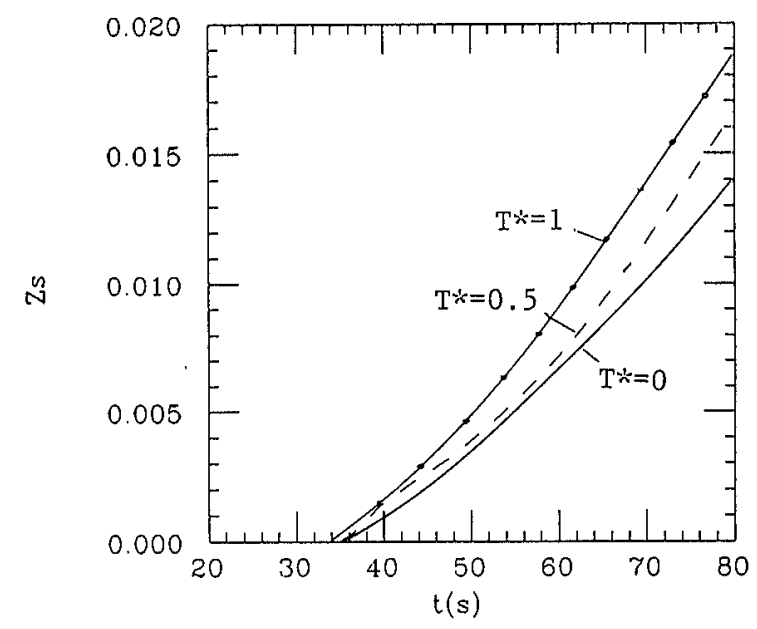

Fig. 13. Evolution of solidification front at point 3 for $B=$ $1 \mathrm{kGauss}$ with $T^{*}$ as a parameter.

contours are essentially flat while in the presence of stirring, these contours have radial variation.

\section{Discussion}

In this paper, we presented computed results describing the evolution of velocity, temperature and solidification of a melt-solid slurry both in the absence and presence of electromagnetic stirring. The main purpose of the work was to combine our recent ability to calculate transport phenomena in melt-solid slurries with the laws of solidification so as to be able to investigate the effect of process parameters on the progress of solidification in a laboratory type apparatus. The micro-modeling issues like nucleation-growth models have not been addressed at this stage. In addition, the time span covered in the calculations corresponds to only a partial solidification of the system. These issues will be investigated further in a subsequent work.

The main contributions of the work described here may be summarized as follows:

(1) We modeled coupled stirring and solidification for the first time, representing the velocity, temperature and solid fraction, albeit for an idealized

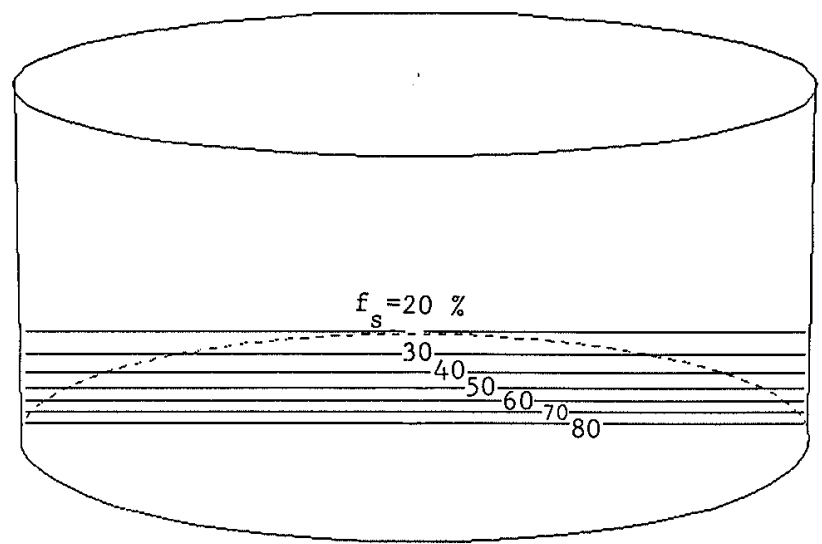

Fig. 14(a). Map of solid fraction after $20 \mathrm{~s}$ in the absence of magnetic field.

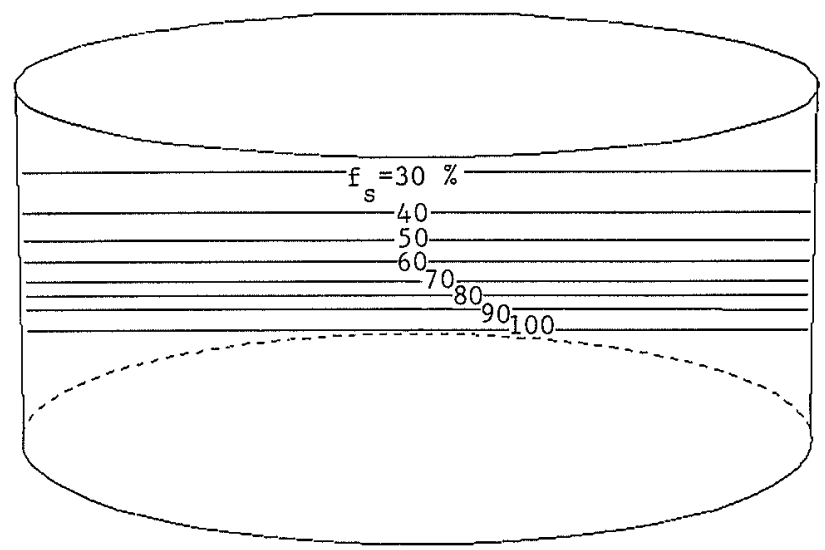

Fig. 14(b). Map of solid fraction after $80 \mathrm{~s}$ in the absence of magnetic field.

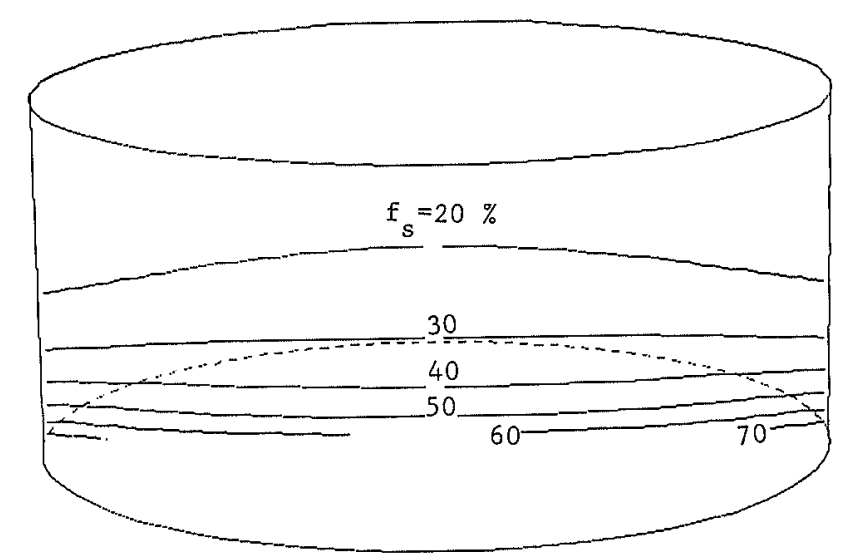

Fig. 14(c). Map of solid fraction after $20 \mathrm{~s}$ for $B=1 \mathrm{kGauss}$.

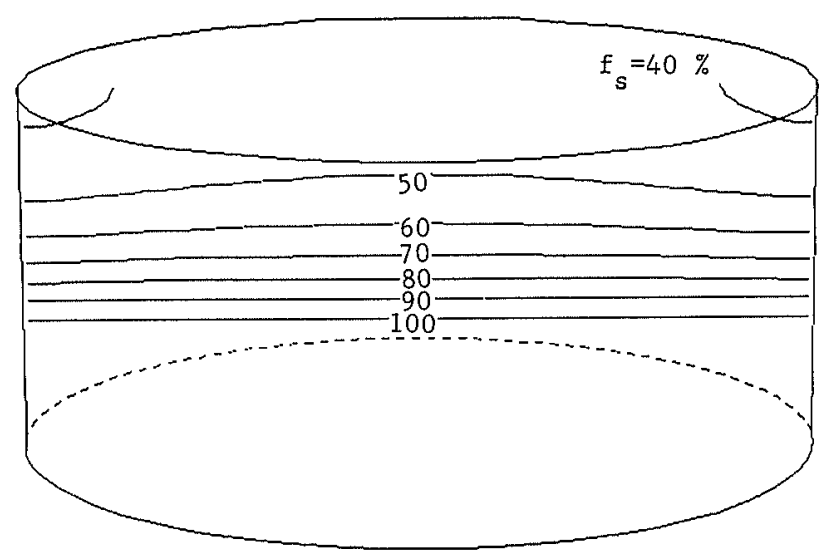

Fig. 14(d). Map of solid fraction after $80 \mathrm{~s}$ for $B=1 \mathrm{kGauss.}$ 
situation.

(2) We have been able to represent, in an approximate fashion, how the temperature and solid fractions evolve with time, as a function of stirring, and the assumption regarding the location of the release of latent heat.

(3) In the model, we allowed for the rheology of the melt-solid suspension.

(4) Perhaps, the most remarkable finding in this work was the fact that the evolution of the temperature and solid fraction profiles was not dramatically affected, either by the stirring rate or by the assumption regarding where the latent heat was being released.

This finding has very important practical consequences which include:

(a) The solidified structures will, of course, be markedly affected by these parameters.

(b) Measuring the temperature progression alone will not give a good indication as to what is happening in the system, unless these temperature measurements are very accurate.

Finally some comments should be made on the simplifying assumptions that have been made in the development of the model, the most important of which were the following:

(1) The postulate of a uniform rotating magnetic field was an idealization, because in most practical cases such field has to be generated by multi-pole magnets, which would give rather more complex field levels; albeit with qualitatively similar behavior to that found here.

(2) In the calculations, joule heating has been neglected. This could become significant, particularly at the higher field levels and may slow down the solidification process.

(3) The rotating melt may exert significant centrifugal forces on the solid particles, causing them to adhere to the solid wall. Such a behavior may favor one of the limiting cases considered, that is the mushy zone fully adhering to the solid wall.

(4) Last, but not least, the consideration of nucleation and growth should be part of a more refined treatment of this problem.

It is hoped to relax these simplifying assumptions as a result of currently ongoing research.

\section{Conclusion}

A mathematical representation has been presented to describe the solidification of a two component, aluminum copper melt, which contains suspended boron carbide particles.

In the model allowance is made for the effect of externally imposed agitation and for the non-Newtonian behavior of the system, due to the presence of the solid particles.

In the development of the nucleation and growth were not considered explicitly, but rather we examined 3 limiting cases, namely when the mushy region fully adhered to the solid phase, when the mushy region was fully suspended in the bulk of the melt and an intermediate between these two.

By running the model we could track the evolution of the temperature profiles in the system and the rate of advancement of the solidification front. This approach is thought to be a necessary intermediate step in representing rheocasting and compocasting processes.

\section{Nomenclature}

$B, B_{0}:$ Magnetic field

$C_{p}, C_{p l}:$ Specific heat

$C_{p \text { eff }}:$ Effective specific heat

$f_{s}:$ Solid fraction plotted

$f_{s 0}$ : Initial solid fraction in melt

$f_{s s}$ : Solid fraction deduced from the enthalpy

$F$ : Electromagnetic force

$h$ : Specific enthalpy

$h_{c}$ : Heat transfer coefficient

$\Delta H:$ Latent heat

$k$ : Thermal conductivity

$L:$ Latent heat of fusion

$L_{r}$ : Latent heat released at solid-melt interface at $T=T_{r}$

$m, n$ : Empirical constants in shear-stress relationship

$p$ : Pressure

$q$ : Heat transfer rate per unit area at cooled plane

$r$ : Radial coordinate

$S_{h}:$ Enthalpy source term

$t$ : Time

T: Temperature

$T_{e}:$ Surrounding temperature

$T_{i}$ : Temperature at surface of cooled plane

$T_{l}:$ Liquidus temperature

$T_{s}$ : Solidus temperature

$T_{r}$ : Temperature at solid-melt interface

$T^{*}$ : Dimensionless temperature (proportion of mushy zone attaching to interface)

$u$ : Velocity component along azimuthal direction

$U:$ Generalised velocity vector

$U_{m}$ : Absolute value of the maximum velocity

$v$ : Velocity component along radial direction

$w$ : Velocity component along axial direction

$z$ : Axial coordinate

$\gamma$ : Rate of deformation tensor

$\mu_{0}$ : Magnetic permeability

$\omega:$ Angular velocity of magnetic field

$\rho$ : Density

$\sigma$ : Electrical conductivity

$\tau:$ Shear stress tensor

$\theta$ : Azimuthal coordinate

\section{Acknowledgments}

This work was sponsored by the National Aeronautics and Space Administration under Research Grant \#NAG-3-808. The authors wish to akcnowledge, with thanks, very helpful discussion with Professor C. Vives of the University of Avignon, France. 


\section{REFERENCES}

1) M. C. Flemings and R. Mehrabian: Trans. Am. Found. Soc., B, 17B (1973), 119.

2) D. A. Melford: Ironmaking Sleelmaking, 7 (1980), 89.

3) J. P. Birat and J. Chone: Ironmaking Steelmaking, 10 (1983), 269.

4) G. Hudault, D. Cheve, J. L. Meyer and J. P. Tartour: "First Experience of Commercial Operation with the CREM Process", Proc. Technical Sessions by the TMS Light Metals Committee at the 118th TMS Annual Meeting, Met. Soc., Warrendale (1989), 769.

5) M. Rappaz: Int. Mater. Rev., 34 (1989), No. 3, 93.

6) P. A. Joly: Ph. D. Thesis, Dept. of Materials Science and Engineering, Massachusetts Institute of Technology, (1974).

7) V. Laxmanan: Ph. D. Thesis, Dept. of Materials Science and Engineering, Massachusetts Institute of Technology, (1979).

8) O.J. Ilegbusi and J. Szekely: Trans. Iron Steel Inst. Jpn., 28 (1988), 97.

9) O. J. Ilegbusi and J. Szekely: ISIJ Int., 29 (1989), 462.

10) V.R. Voller, M. Cross and N. C. Markatos: Int. J. Numerical Methods in Engineering, 24 (1987), 271.

11) V. R. Voller and G. Prakash: Int. J. Heat Mass Transf., 30 (1987), 1709.

12) V. Stanek and J. Szekely: Metall. Trans. B., 7B (1976), 619.

13) K. H. Spitzer, M. Dubke and K. Schwerdtfeger: Metall. Trans. B., 17B (1986), 119.

14) D. B. Spalding: Mathematics and Computers in Simulation, 13 (1981), 267. 